\title{
Effect of pidotimod on growth performance, immune function, intestinal epithelial barriers and microbiota of piglets
}

\author{
Z. Zeng ${ }^{1}$, L. Tang ${ }^{1}$, Y. Zhou ${ }^{1}$, B. Wang ${ }^{1}$, Q. Wang ${ }^{1}$, P. Zou' ${ }^{1}$, X. Zhan ${ }^{1}$, L. Fu $u^{1,2,3}$ and W. Li ${ }^{1,3}$ \\ ${ }^{1}$ Zhejiang University, College of Animal Sciences, Institute of Animal Nutrition and Feed Sciences, Key Laboratory of Molecular \\ Animal Nutrition of the Ministry of Education, National Engineering Laboratory of Biological Feed Safety and Pollution Prevention \\ and Control, Key Laboratory of Animal Feed and Nutrition of Zhejiang Province, Hangzhou 310058, Zhejiang Province, China \\ ${ }^{2}$ Department of General Surgery, Chun'an First People's Hospital (Zhejiang Provincial People's Hospital Chun'an Branch), \\ Hangzhou 311700, Zhejiang Province, China
}

KEY WORDS: cytokines, intestinal microorganisms, jejunum, pidotimod, piglets

Received: 22 December 2020

Revised: $\quad 5$ March 2021

Accepted: $\quad 8$ March 2021

${ }^{3}$ Corresponding author:

e-mail: fuluoqin@gmail.com,wfli@zju.edu.cn

\begin{abstract}
Pidotimod (PTD) is a synthetic immunomodulatory dipeptide widely used for immune regulation. This study aimed to investigate the effect of PTD on growth performance, immune function, intestinal epithelial barriers and microbiota in piglets. In total, 120 piglets with a weight of $25.26 \pm 5.96 \mathrm{~kg}$ and genetic makeup were selected and randomly divided into 4 groups with 3 replicates per group and 10 piglets per replicate. All piglets were weighed, and from 3 piglets from each replicate ( 9 piglets per group) tissues were collected. Piglets were fed for 44 days basal diet alone or supplemented with 50,100 or $200 \mathrm{mg} / \mathrm{kg}$ PTD. It was found that the PTD addition increased the average daily weight gain of piglets $(P<0.05$ and $P<0.01$ for dose 50 and $100 \mathrm{mg} / \mathrm{kg}$, respectively). So, for further studies only dose $50 \mathrm{mg} / \mathrm{kg}$ PTD was used. It was found that such dose decreased serum levels of interleukin (IL)-6 $(P<0.05)$ and interferon (IFN)-y $(P<0.01)$, but increased antiinflammatory cytokine IL-10 level $(P<0.05)$. Transmission electron micrographs further revealed that supplementation with PTD at a dose of $50 \mathrm{mg} / \mathrm{kg}$ enhanced intercellular connectivity, characterized by longer tight junction (TJ) proteins, thicker adhesive tapes (AB) and desmosomes (D) in the jejunum. This phenomenon was validated by the up-regulated protein expression of occludin and ZO-1 in the jejunum. Furthermore, supplementation with PTD at a dose of $50 \mathrm{mg} / \mathrm{kg}$ altered the a diversity of intestinal microbiota in piglets (Observed_species, Chao1, PD_whole_tree, Ace). Particularly, the abundance of Spirochaetes, Verrucomicrobia, Succinispira and Treponema was decreased, but that of Lactobacillus and Collinsella was increased. Overall, dietary supplementation with $50 \mathrm{mg} / \mathrm{kg}$ PTD improves the growth performance of piglets by enhancing immune function, intestinal epithelial barrier and regulating microbiota composition.
\end{abstract}

\section{Introduction}

Pidotimod (PTD) is a synthetic dipeptide with biological immunomodulatory and anti-tumour properties (Riboldi et al., 2009). Due to the immunomodulatory activity, PTD is able both to improve the clinical conditions of patients and to enhance and stimulate their immune cell functions, thereby exerting adaptive and innate immune functions (Ferrario et al., 2015). In comparison with traditional immunomodulators, PTD is very safe, robust and stable (Tian and Zeng, 2005). It was shown that 
a higher PTD dose $(800 \mathrm{mg} / \mathrm{kg}$ oral for rats and $600 \mathrm{mg} / \mathrm{kg}$ oral for dogs) induced no adverse reactions in these animals (Coppi et al., 1994). Moreover, the bioavailability of intramuscularly injected PTD was $100 \%$ but decreased to just $27 \%$ when administered orally (Coppi and Silingardi, 1994). Due to a good thermal stability, it can be administered directly without coating (Fu et al., 2016).

Currently, the immune dipeptide is mainly applied in preventing and treating repeated respiratory and urinary tract infections as well as acute and chronic bronchitis infections both in children and adults (Mahashur et al., 2019). In vivo and in vitro experiments revealed that PTD can significantly enhance the functions of natural killer (NK) cells, the phagocytic activity of monocytes and the chemotactic roles of neutrophils (Migliorati et al., 1992; 1994). Besides, it can restore the normal expression of immuno-modulated peritoneal macrophages (Capsoni et al., 1992). Pidotimod induces the maturation of human peripheral blood dendritic cells (DC) and up-regulates the expression of human leukocyte DR antigen (HLA-DR) and co-stimulatory molecules such as CD83 and CD86 (Puggioni et al., 2019). Moreover, it promotes the release of several pro-inflammatory molecules such as monocyte chemoattractant protein-1 (MCP-1) and tumour necrosis factor (TNF)- $\alpha$, thereby promoting the proliferation and differentiation of $\mathrm{T}$ cells into Th1 phenotype (Giagulli et al., 2009). In addition, in the in vitro study it was demonstrated that PTD can inhibit the proliferation as well as induce apoptotic death of YAC-1 tumour cells (Migliorati et al., 1993).

In recent years, the increase in intensive farming of highbred animals has resulted in a frequent outbreak of diseases in the farms (Amadori and Zanotti, 2016). This raises the cost of production and lowers the quality of animal products. Therefore, there is an unmet need to develop sustainable disease prevention strategies. It was noted that oral administration of $1 \mathrm{~g} / \mathrm{l}$ of PTD increased the production of antibodies in broilers, which effectively enhanced resistance of birds against lethal Newcastle disease virus (Li et al., 2016); it was further confirmed by $\mathrm{Qu}$ et al. (2017). However, effects of PTD in pigs remained to be validated. Meanwhile, piglet intestinal health is one of the most important factors affecting pigs under intensive production systems. It was reported that in China, the average annual mortality and morbidity of piglets below $30 \mathrm{~kg}$ is as high as 10.3 and $46.5 \%$, respectively (Zhong et al., 2005). This underlines the role of intestinal health and diseases limited the expansion of the pig industry
(Zhong et al., 2005). Although piglet intestinal health is often determined by the interaction of multiple factors, intestinal microbes have been shown to be the most fundamental factor. This is attributed to formation of a well-developed immune system promotion (Fulde and Hornef, 2014) and provides sufficient resistance against pathogens (Kamada et al., 2013). It was observed that intestinal microbes can activate regulatory $\mathrm{T}$ cells (Treg) to secrete cytokines and inhibit the overgrowth of pathogens (Wang et al., 2015). It is worth mentioning that piglet intestinal health can be improved by improving the immune function in animals. Unfortunately, the application of PTD in pig production is only scarcely reported. Consequently, we explored the effect of PTD on the growth performance, immune function as well as intestinal epithelial barriers and microbiota composition in piglets.

\section{Material and methods}

\section{Reagents}

Pidotimod was synthesized by Shandong Qilu Pharmaceutical Co., Ltd. Production (Jinan, China); batch number: 201009001. Different amounts of PTD were mixed with the feed directly before the experiment.

\section{Animals, diet and management}

All procedures of this study were in accordane with the Chinese guidelines for animal welfare. All procedures were approved by the Institutional Animal Care and Use Committee of Zhejiang University (Zhejiang Province, China), permission number: ZJU20160416.

In total, 120 healthy piglets (Duroc $\times$ Landrace $\times$ Yorkshire) with a weight of $25.26 \pm 5.96 \mathrm{~kg}$ were fed the same basal diet in a closed pigsty with consistent environmental conditions and feeding management at a suitable temperature $\left(\sim 23-25^{\circ} \mathrm{C}\right)$. To compare the effects of different doses of PTD on the growth performance, piglets were randomly divided into 4 groups: control group (CON) was fed basal diet, and treatments 1 to 3 were supplemented with 50,100 and $200 \mathrm{mg} / \mathrm{kg}$ of PTD, for 44 days. Each group has 3 replicates, 10 piglets per replicate, 5 barrows and 5 gilts. Feed and water were given $a d$ libitum. Basal diet was formulated according to the nutritional requirements of the National Research Council (NRC, 2012). Its nutrient composition is shown in Table 1. Piglets were weighed at the beginning and the end of the trial. Before weighing, 
Table 1. Composition and nutrient level of the basal diet, $\%$ as fed basis

\begin{tabular}{|c|c|c|c|}
\hline Ingredients & Content, $\%$ & Nutrient levels ${ }^{2}$ & Content, $\%$ \\
\hline Maize & 61.25 & $\begin{array}{l}\text { Digestible energy, } \\
\mathrm{MJ} / \mathrm{kg}\end{array}$ & 14.11 \\
\hline $\begin{array}{l}\text { Soyabean meal ( } 44 \% \\
\text { crude protein }(C P))\end{array}$ & 15.79 & Calcium & 0.80 \\
\hline $\begin{array}{l}\text { Extruded soyabean } \\
(36 \% \mathrm{CP})\end{array}$ & 10.00 & Total phosphorus & 0.63 \\
\hline $\begin{array}{l}\text { Imported fish meal } \\
(65 \% \mathrm{CP})\end{array}$ & 5.00 & $\begin{array}{l}\text { Available } \\
\text { phosphorus }\end{array}$ & 0.40 \\
\hline Wheat bran & 3.00 & Lysine & 1.15 \\
\hline Soyabean oil & 1.74 & $\begin{array}{l}\text { Methionine + } \\
\text { Cysteine }\end{array}$ & 0.67 \\
\hline Premix ${ }^{1}$ & 1.00 & Threonine & 0.77 \\
\hline Limestone & 0.98 & Tryptophan & 0.22 \\
\hline $\mathrm{CaHPO}_{4}$ & 0.78 & $\mathrm{CP}$ & 19.00 \\
\hline Salt & 0.37 & Crude ether extract & 7.26 \\
\hline \multirow[t]{2}{*}{ Lysine- $\mathrm{HCl}$} & 0.09 & Crude fibre & 2.30 \\
\hline & & Ash & 6.27 \\
\hline
\end{tabular}

${ }^{1}$ provided per kg of diet: $\mathrm{mg}: \mathrm{Zn}(\mathrm{ZnO}) 50, \mathrm{Cu}\left(\mathrm{CuSO}_{4}\right)$ 20, $\mathrm{Mn}(\mathrm{MnO})$ 55, $\mathrm{Fe}\left(\mathrm{FeSO}_{4}\right)$ 100, I (KI) 1, $\mathrm{Co}\left(\mathrm{CoSO}_{4}\right)$ 2, Se $\left(\mathrm{Na}_{2} \mathrm{SeO}_{3}\right)$ 0.3; vitamin $B_{1} 2$, vitamin $B_{2} 4$, pantothenic acid 15 , vitamin $B_{6} 10$, vitamin $B_{12} 0.05$, vitamin PP 30 , folic acid 2, vitamin $\mathrm{K}_{3} 1.5$, biotin 0.2 , choline chloride 800, vitamin C 100; IU: vitamin A 8255, vitamin $D_{3} 2000$, vitamin E 40; ${ }^{2}$ calculated values

pigs were fasted for $12 \mathrm{~h}$ and coaxed for $10 \mathrm{~min}$ and weighed after excretion of urine to calculate the daily weight gain. The daily feed intake was also recorded. According to feed intake and daily weight gain, the feed conversion ratio was calculated as:

feed conversion ratio $=$ total feed consumption $(\mathrm{kg})$ / total weight gain $(\mathrm{kg})$.

Based on the analysis of daily weight gain and feed conversion ratio, the supplementation with PTD at a dose of $50 \mathrm{mg} / \mathrm{kg}$ resulted in the best growth performance among the three groups, and so was used in the subsequent investigations.

\section{Sample collection}

At the beginning and the end of the feeding test, all piglets were weighed after deprivation of feed overnight. Then, 3 piglets from each replicate (9 piglets per group) were electrically stunned, exsanguinated, and scalded to enable to collect tissues. The spleen and liver were collected, and the spleen was weighed after removing the adherent fatty tissue. The spleen index was calculated as:

spleen index $(\mathrm{g} / \mathrm{kg})=$ spleen weight $/$ live weight before slaughter.

The serum was collected for the ELISA test. Caecum contents were collected, stored at $-80{ }^{\circ} \mathrm{C}$ for DNA extraction. The mid-jejunum segments were fixed in $2.5 \%$ glutaraldehyde, rinsed with $0.1 \mathrm{M}, \mathrm{pH} 7.0$ phosphate buffer, and next fixed with
$1 \% \mathrm{OsO}_{4}$ buffer for transmission electron microscopy (TEM). Spleen and liver samples were fixed in 4\% paraformaldehyde for hematoxylin and eosin (H\&E) staining. The jejunum mucosa was gently scraped, stored at $-80^{\circ} \mathrm{C}$ for Western blot analysis.

\section{Hematoxylin and eosin (H\&E) staining}

Liver and spleen samples of piglets fed diet supplemented with PTD at a dose of $50 \mathrm{mg} / \mathrm{kg}$ were fixed in $4 \%$ paraformaldehyde, embedded in paraffin, sliced, dehydrated, and stained with hematoxylin and eosin. Images were captured using an Olympus microsystem (Tokyo, Japan).

\section{Transmission electron microscopy (TEM)}

Specimens of piglets fed diet supplemented with PTD at a dose of $50 \mathrm{mg} / \mathrm{kg}$ were first fixed with $2.5 \%$ glutaraldehyde and then fixed with $1 \% \mathrm{OsO}_{4}$ buffer. The sample was then dehydrated with a gradient of ethanol solution. After infiltration and embedding, middle jejunum samples were ultrathin sectioned and observed under a Hitachi Model H-7650 transmission electron microscope (Tokyo, Japan).

\section{Serum cytokines}

Blood samples from piglets fed diet supplemented with PTD at a dose of $50 \mathrm{mg} / \mathrm{kg}$ were collected from the vena cava anterior and then centrifuged for $10 \mathrm{~min}$ at $4{ }^{\circ} \mathrm{C}(3000 \mathrm{~g}$, Centrifuge 5804R, Eppendorf, Hamburg, Germany) to obtained serum samples. Levels of inflammatory factors (interleukin (IL)-6, IL-8, tumor necrosis factor (TNF)- $\alpha$ ), Th1 cytokines (IL-12 p40, interferon (IFN)- $\gamma$ ), Th2 cytokines (IL-4), anti-virus-related cytokines (IFN- $\alpha$ ) and anti-inflammatory factors (IL-10) in serum were determined by porcine enzyme-linked immunosorbent assay (ELISA) kits according to the manufacturer's instructions (eBioscience, Santa Clara, CA, USA). The standard curves were made according to the instructions $\left(\mathrm{R}^{2}>0.99\right)$, concentrations of cytokines were then calculated from the standard curves.

\section{Western blotting}

The jejunum mucosal samples collected from piglets fed diet supplemented with PTD at a dose of $50 \mathrm{mg} / \mathrm{kg}$ were collected and homogenized in radio-immunoprecipitation assay (RIPA) buffer (Beyotime, Shanghai, China). The protein concentration was determined using a BCA kit (Beyotime, Shanghai, China). After denaturation, proteins from each sample were separated by sodium dodecyl sulphate (SDS)-polyacrylamide gel electrophoresis and then transferred to nitrocellulose membranes 
(Roche, Mannheim, Germany). The membranes were blocked by $5 \%$ skimmed milk then incubated with primary antibody overnight at $4{ }^{\circ} \mathrm{C}$. Primary antibodies occludin, claudin-1 and ZO-1 (ab222691, ab129119, ab214228) were purchased from Abcam (Cambridge, UK), while $\beta$-actin antibody (AA128) was purchased from Beyotime (Shanghai, China). After incubation with secondary antibody (A0208, A0216, Beyotime, Shanghai, China) for $1 \mathrm{~h}$ at room temperature, the immunoreactive bands were detected by an ECL system (Tanon, Shanghai, China). The relative band density was determined by an ImageJ 1.52 software (Schneider et al., 2012).

\section{Microbial analysis}

The caecal microbial genomic DNA of piglets fed diet supplemented with PTD at a dose of $50 \mathrm{mg} / \mathrm{kg}$ was extracted by a TIANamp Stool DNA Kit (Tiangen, Beijing, China) following the manufacturer's instructions. The V3-V4 hypervariable region of the $16 \mathrm{~S}$ rDNA was amplified using the primer pair 341F (5'-CCTACGGGNGGCWGCAG-3') and 805R (5'-GACTACHVGGGTATCTAATCC-3'). The PCR procedure was set according to our previous study (Xu et al., 2018). Then the sequencing was performed by the MiSeq platform (Illumina Inc., San Diego, CA, USA). Sequences were filtered and clustered into operational taxonomic units (OTU) at $97 \%$ similarity by a QIIME software (ver. 1.9.1) (Caporaso et al., 2010). Bacterial OTU representative sequences were assigned to a taxonomic lineage by Ribosomal Database Project (RDP) classifier based on the Greengenes database (13_8 release). Alpha diversity and beta diversity between samples were also analyzed by QIIME software using 'alpha_diversity.py' and 'beta_diversity through_plots.py'. Beta diversity was displayed by principal components analysis (PCA) using the 'ggplot2' package of R software and permutational multivariate analysis of variance (PERMANOVA) was calculated to determine significant differences in the microbial community using the 'vegan' package (based on the Bray-Curtis distance matrices).

\section{Statistical analysis}

Growth performance data were analyzed by a one-way ANOVA followed by the Tukey's test. Others were analyzed for 2 groups by a two-tailed t-test using SPSS 22 (SPSS Inc., Chicago, IL, USA) for Windows and results were expressed as mean \pm standard deviation (SD). Data were significantly different when $P<0.05\left(^{*}\right)$ and $P<0.01(* *)$. Data were visualized by an Origin 9.0 software (Origin Lab Corporation, Northampton, MA, USA).

\section{Results}

\section{Growth performance}

As shown in Table 2, in comparison with control, PTD supplementation significantly increased the average daily weight gain of piglets $(P<0.05$ and $P<0.01$ for dose of 50 and $100 \mathrm{mg} / \mathrm{kg}$, respectively).

Table 2. Effect of pidotimod on the growth performance of piglets

\begin{tabular}{|c|c|c|c|c|}
\hline \multirow{2}{*}{ Indices } & \multirow{2}{*}{ Control } & \multicolumn{3}{|c|}{ Pidotimod, mg/kg } \\
\hline & & 50 & 100 & 200 \\
\hline $\begin{array}{l}\text { Initial body } \\
\text { weight, kg }\end{array}$ & $24.60 \pm 5.48$ & $25.15 \pm 5.19$ & $24.48 \pm 3.11$ & $26.82 \pm 8.86$ \\
\hline $\begin{array}{l}\text { Final body } \\
\text { weight, kg }\end{array}$ & $47.74 \pm 5.83$ & $51.93 \pm 5.17$ & $52.08 \pm 4.2$ & $50.5 \pm 8.41$ \\
\hline $\begin{array}{l}\text { Average daily } \\
\text { feed intake, } \\
\text { kg/day }\end{array}$ & $1.21 \pm 0.11$ & $1.28 \pm 0.03$ & $1.40 \pm 0.15$ & $1.37 \pm 0.21$ \\
\hline $\begin{array}{l}\text { Average daily } \\
\text { gain, kg/day }\end{array}$ & $0.55 \pm 0.01^{a b}$ & $0.61 \pm 0.01^{a}$ & $0.64 \pm 0.03^{a}$ & $0.5 \pm 0.0^{2 b}$ \\
\hline $\begin{array}{l}\text { Feed } \\
\text { conversion } \\
\text { ratio }\end{array}$ & $2.19 \pm 0.19$ & $2.10 \pm 0.07$ & $2.20 \pm 0.15$ & $2.50 \pm 0.45$ \\
\hline
\end{tabular}

values are mean $\pm S D$; data were analyzed by a one-way ANOVA followed by Tukey test ( $n=30$ in each group), ${ }^{\text {ab }}-$ values with different superscripts for each parameter are significantly different at $P<0.05$

Comparatively, the lower feed conversion ratio was stated in piglets fed diet supplemented with PTD at a dose of $50 \mathrm{mg} / \mathrm{kg}$ conferred the greatest feed conversion performance $(P>0.05)$, without inducing apparent undesirable side effects on the spleen and liver (Figure 1). Moreover, there were no differences in spleen index between the control and the PTD group. Accordingly, PTD dose of $50 \mathrm{mg} / \mathrm{kg}$ was used in the subsequent investigations.

\section{Cytokines level}

As shown in Figure 2, in comparison with the control group, supplementation with PTD at a dose of $50 \mathrm{mg} / \mathrm{kg}$ significantly reduced serum levels of pro-inflammatory cytokine IL-6 $(P=0.0415)$, IFN- $\gamma$ $(P=0.0041)$, and had no effects on TNF- $\alpha$, IL-8, IL-4, IL-12 p40 and IFN- $\alpha$ cytokines $(P>0.05)$. However, it remarkably up-regulated serum levels of IL-10 $(P=0.0374)$.

\section{TEM observation and expression of tight junction proteins in the jejunum of piglets}

Transmission electron microscopy further revealed that in comparison with the control group, PTD dose of $50 \mathrm{mg} / \mathrm{kg}$ increased the intercellular connectivity, characterized by longer tight junction (TJ) proteins as well as denser adhesive tapes $(\mathrm{AB})$ and desmosomes (D) in jejunal cells of piglets 

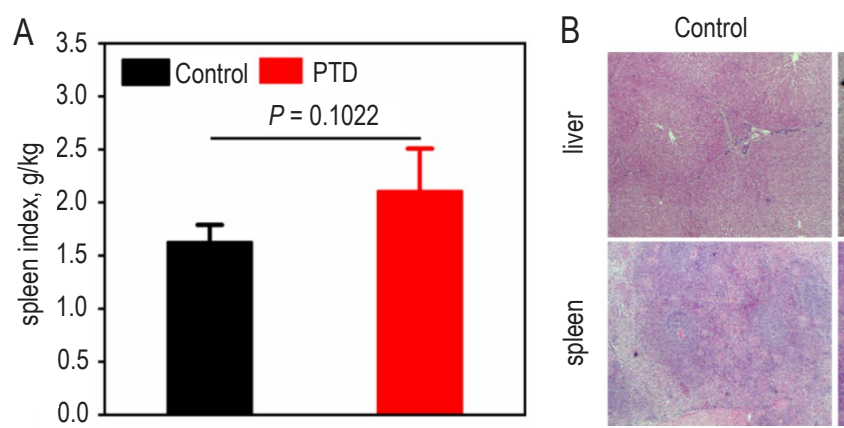

PTD

Figure 1. Effects of pidotimod on spleen index $(A)$ and histopathology of organs in piglets $(B)$

Control group was fed basal diet, pidotimod (PTD) group was fed basal diet supplemented with PTD at a dose of $50 \mathrm{mg} / \mathrm{kg}$; (A): no significant variation has been observed on piglet spleen index: spleen index $(\mathrm{g} / \mathrm{kg})=$ spleen weight / live weight before slaughter, values are mean $\pm \mathrm{SD}$ $(n=9)$; (B): hematoxylin and eosin staining, 40x magnification, scale bar: $200 \mu \mathrm{m}$; the histomorphology of organs is the same between the two groups; the structure of the liver and spleen is clear, and there is no obvious chronic inflammatory cell infiltration in the portal area.
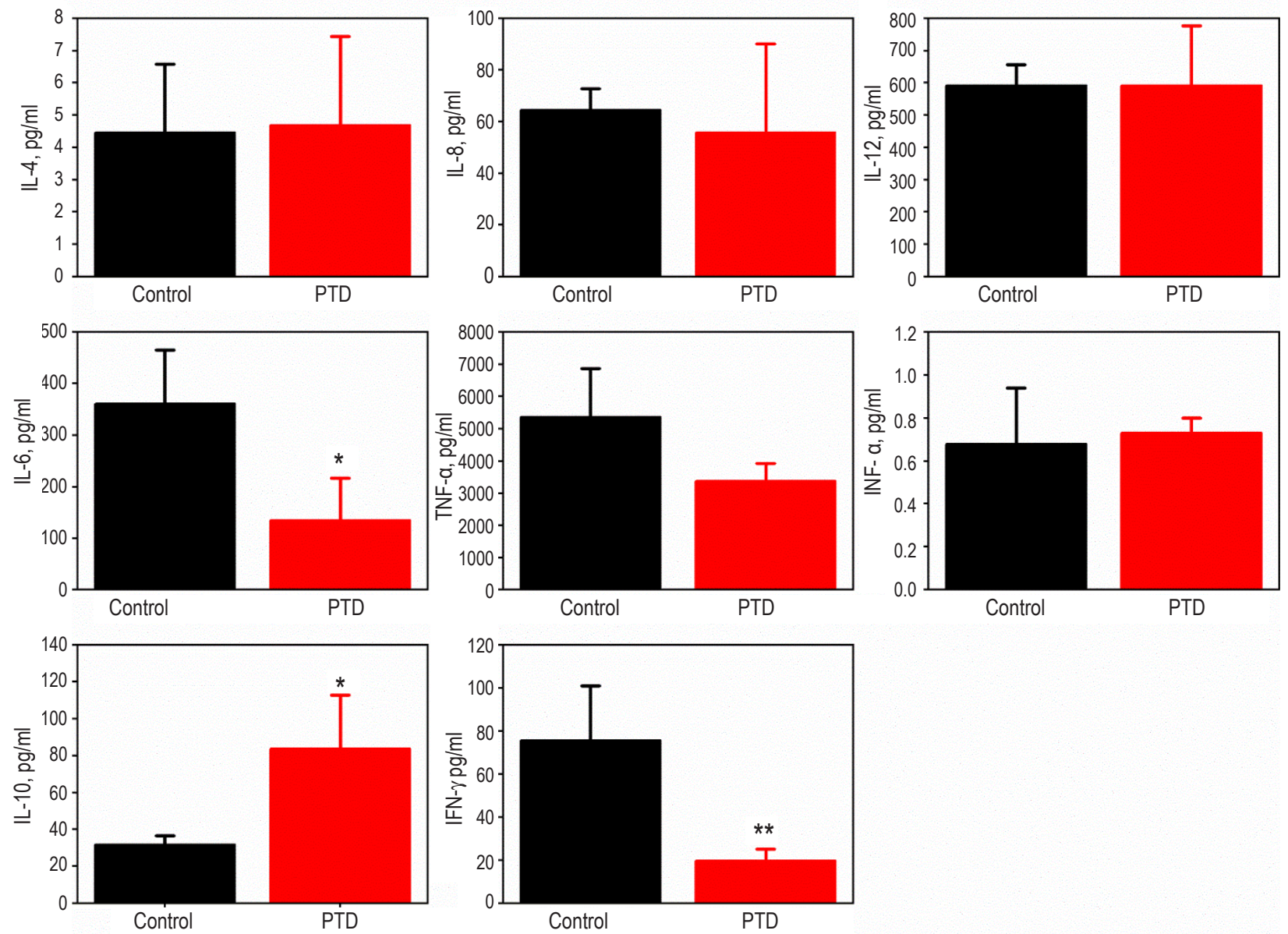

Figure 2. Effects of pidotimod on serum cytokines levels in piglets

Control group was fed basal diet, pidotimod group (PTD) was fed basal diet supplemented with PTD at a dose of $50 \mathrm{mg} / \mathrm{kg}$; values are mean \pm SD ( $=9$ ); * $-P<0.05,{ }^{* *}-P<0.01$; IL-4 - interleukin-4, IL-8 - interleukin-8, IL-12 - interleukin-12, IL-6 - interleukin-6, TNF-a - tumor necrosis factor- $a$, IFN- $\alpha$ - interferon- $\alpha$, IL-10 - interleukin-10, IFN- $y-$ interferon- $y$.

(Figure 3). Western blot analysis further revealed that PTD dose of $50 \mathrm{mg} / \mathrm{kg}$ supplemented to piglets diet up-regulated the protein expression of occludin $(P<0.05)$ and ZO-1 $(P<0.05)$ in jejunal mucosa (Figure 4).

\section{Pidotimod influence on intestinal microbiota}

Alpha diversity analysis showed that PTD supplemented at a dose of $50 \mathrm{mg} / \mathrm{kg}$ significantly $(P<0.05)$ decreased the Observed_species, Chao1, PD_whole_tree and Ace indices (Figure 5). Principal 

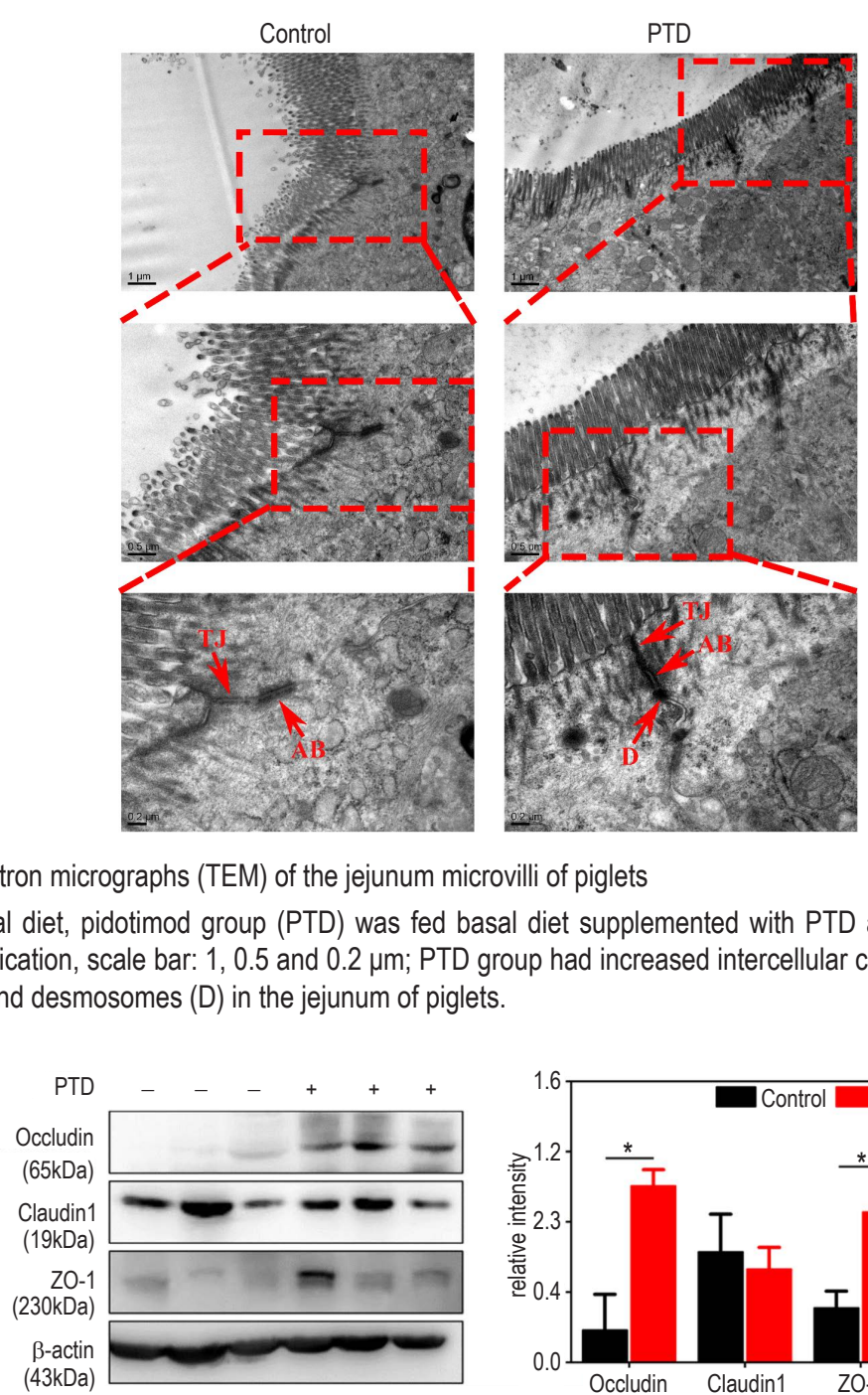

Figure 3. Transmission electron micrographs (TEM) of the jejunum microvilli of piglets

Control group was fed basal diet, pidotimod group (PTD) was fed basal diet supplemented with PTD at a dose of $50 \mathrm{mg} / \mathrm{kg}$. Images were observed at 30,000x magnification, scale bar: 1, 0.5 and $0.2 \mu \mathrm{m}$; PTD group had increased intercellular connectivity, longer tight junctions (TJ), denser adhesive belt (AB) and desmosomes (D) in the jejunum of piglets.

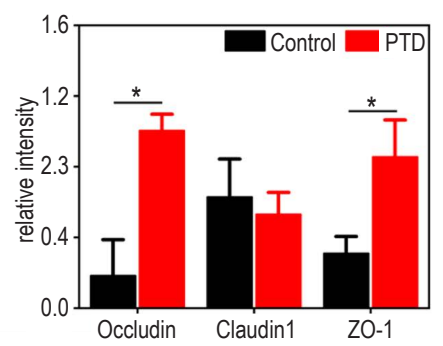

Figure 4. Effects of pidotimod on the expression of tight junction proteins in jejunal mucosa of piglets

Control group was fed basal diet, pidotimod group (PTD) was fed basal diet supplemented with PTD at a dose of $50 \mathrm{mg} / \mathrm{kg}$. Protein lysates from jejunal mucosa were examined by Western blot for occludin, claudin 1 and ZO-1 protein levels. The ratio of Occludin, Claudin 1 and ZO-1 to $\beta$-actin was analyzed using ImageJ software (Schneider et al., 2012); values are mean $\pm \operatorname{SD}(n=3) ;{ }^{*}-P<0.05$.
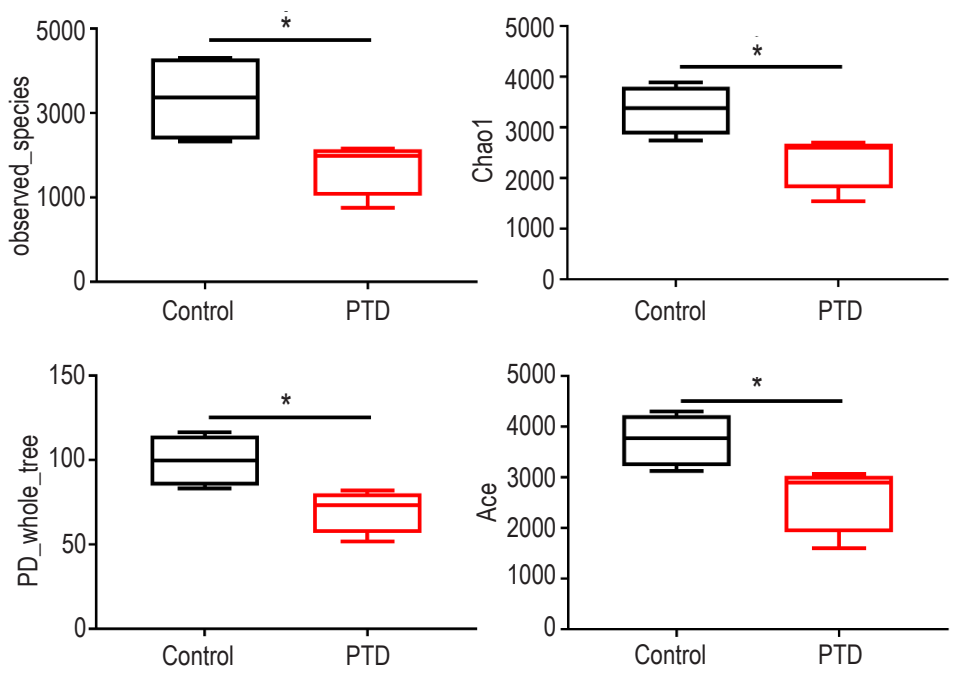

Figure 5. a diversity estimates for the samples

Control group was fed basal diet, pidotimod group (PTD) was fed basal diet supplemented with PTD at a dose of $50 \mathrm{mg} / \mathrm{kg} ;{ }^{*} P<0.05 ; \mathrm{n}=4$. 


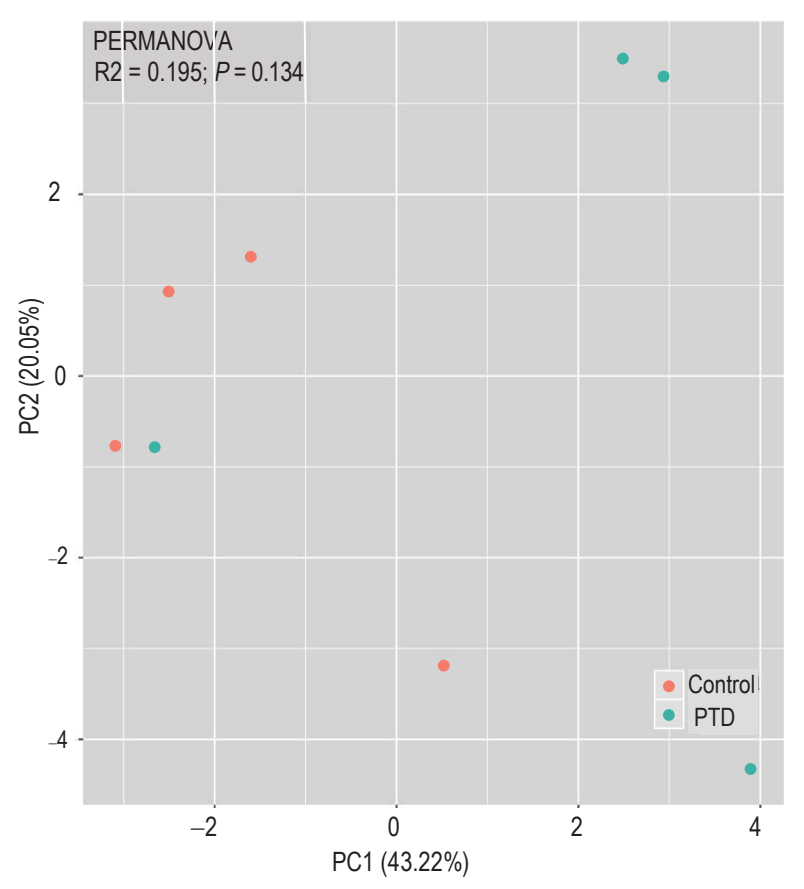

Figure 6. Principal component analysis (PCA) of intestinal microbial communities between the two groups based on Bray-Curtis distance Control group was fed basal diet, pidotimod group (PTD) was fed basal diet supplemented with PTD at a dose of $50 \mathrm{mg} / \mathrm{kg} ; \mathrm{n}=4$.

component analysis (PCA) based on Bray-Curtis distance revealed that PDT had no significant effect on the beta diversity of microbial communities $(\mathrm{R} 2=0.195, P=0.134)$ (Figure 6).

In total, 24 phyla were identified in both control and PTD groups, there were no difference in the microbe number of the control group and PTD group at the phylum level. Firmicutes and Bacteroidetes are the most dominant phyla in the caecum of piglets, accounting for more than 90\% (Figure 7) of the total microbiota. LDA Effect Size (LEfSe) analysis (Figure 8) showed that Bacilli, Lactobacillales,
Streptococcus and Streptococcaceae were the most abundant taxa in the experimental group, converse to taxa Spirochaetales, Spirochaetaceae, Treponema, Peptococcaceae and $\mathrm{rc}_{4} 4$ in the control group.

In comparison with control diet, PTD decreased the relative abundance of Spirochaetes $(P=0.051)$ and Verrucomicrobia $(P=0.059)$ at phyla level and that of Succinispira $(P=0.024)$ and Treponema $(P=0.048)$ at the genus level. However, there was a substantial increase in the abundance of Lactobacillus $(P=0.082)$ and Clinsella $(P=0.074)$ (Figure 9) at the genus level.

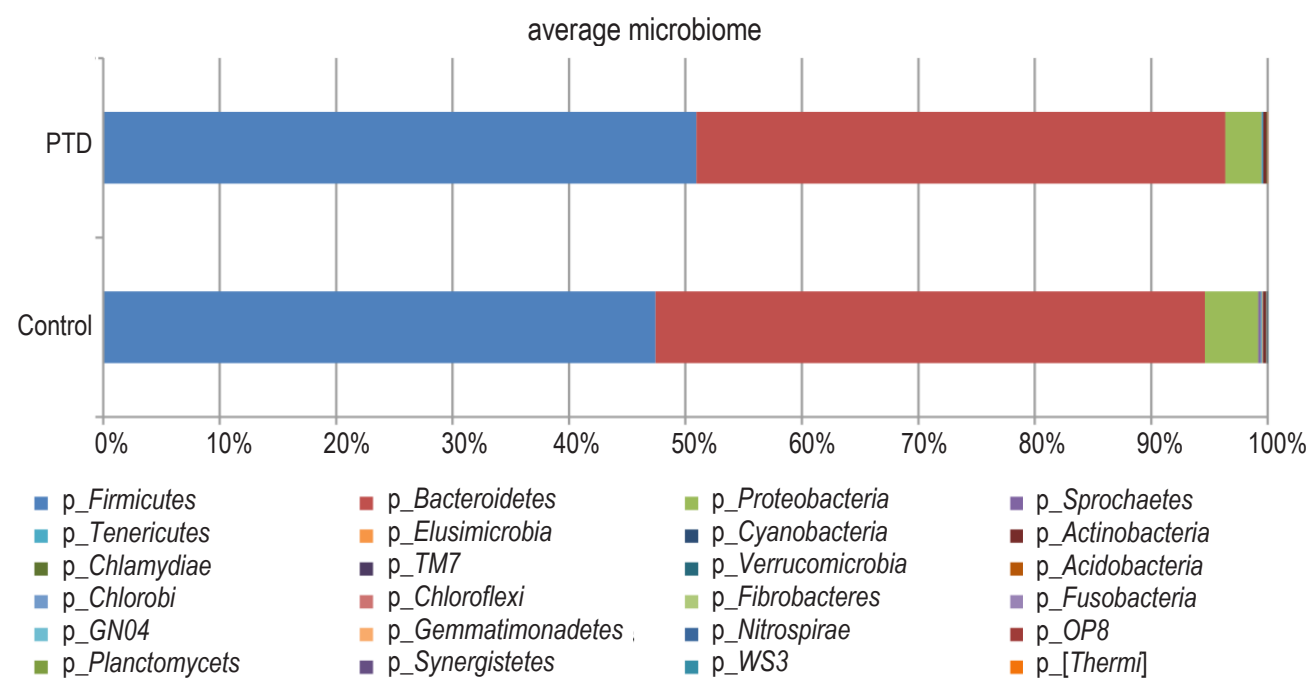

Figure 7. Phylum distributions of intestinal microbiota

Control group was fed basal diet, pidotimod group (PTD) was fed basal diet supplemented with PTD at a dose of $50 \mathrm{mg} / \mathrm{kg} ; \mathrm{n}=4$. 


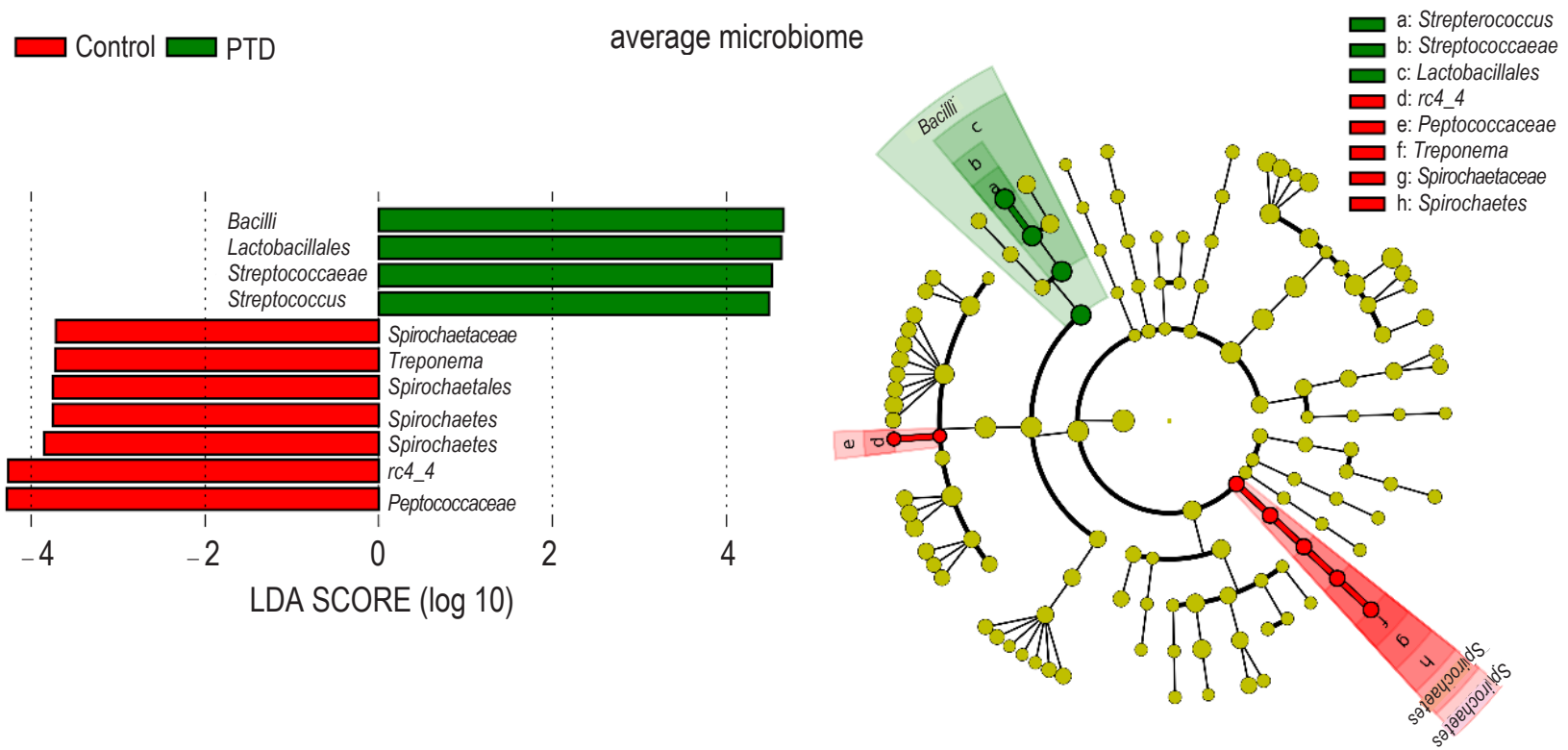

Figure 8. LEfSe analysis of intestinal microbiota
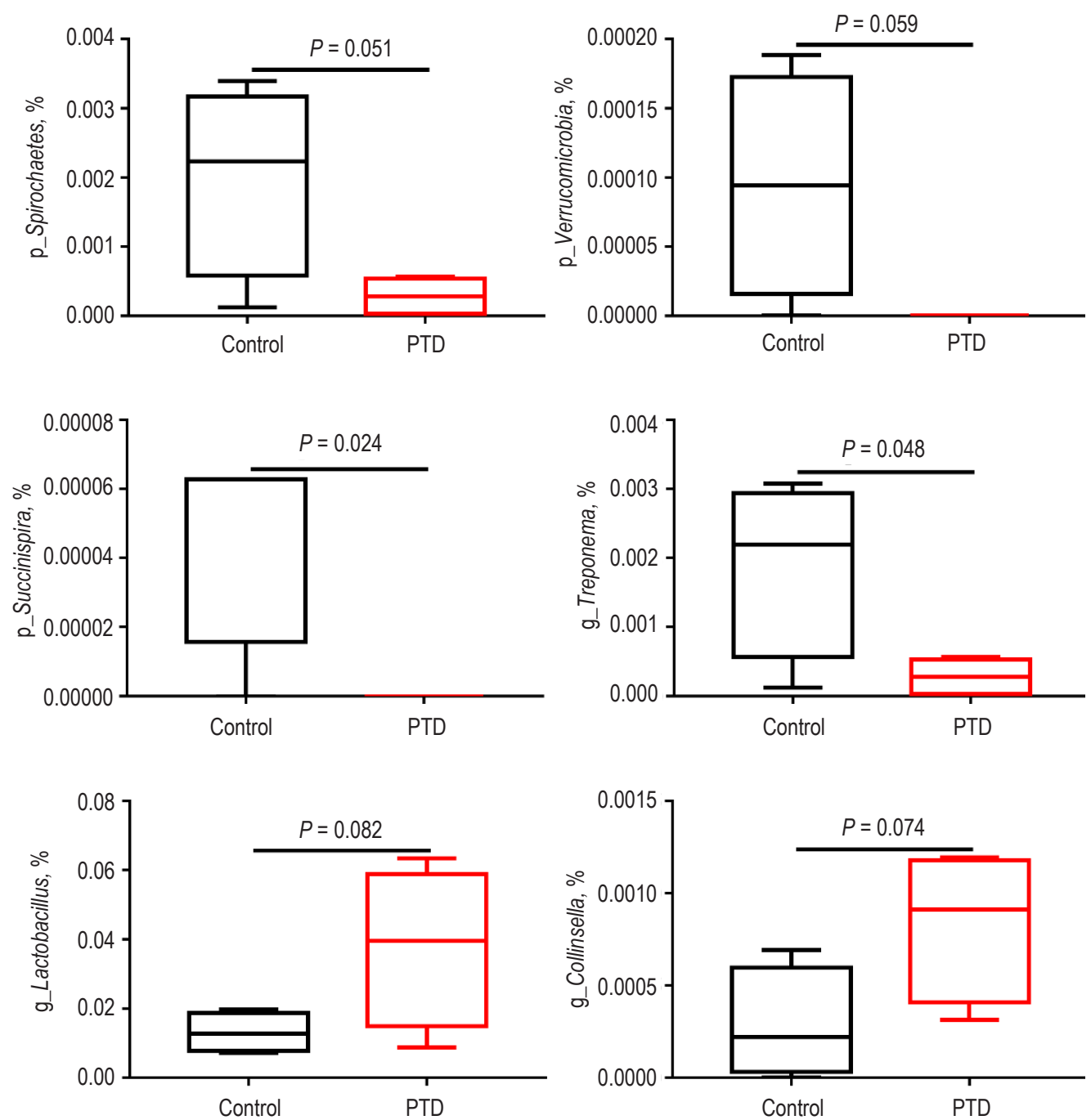

Figure 9. Differentially abundant features between control and pidotimod groups at the phylum and genus levels Control group was fed basal diet, pidotimod group (PTD) was fed basal diet supplemented with PTD at a dose of $50 \mathrm{mg} / \mathrm{kg}$. 


\section{Discussion}

Immunomodulators can improve the growth and immune performance of weaned piglets, thereby enhancing their disease resistance capacity (Zhong et al., 2016). At the same time, it was shown that PTD did not induce teratogenic effects and reproductive toxicity in animals (Caramia et al., 1994; Clemente et al., 1994). In this study, in comparison with the control group, the spleen index of piglets from group fed diet supplemented with PTD at a dose of $50 \mathrm{mg} / \mathrm{kg}$ did not differ significantly and there were no undesirable side effects in the spleen and liver according to H\&E staining. The spleen is an important immune organ, and the index of the spleen was found to decrease in the case of injury (Wu et al., 2020). Our finding provided strong evidence for the safety of PTD. Moreover, based on the average daily weight gain that was measured, PTD accelerated the growth of piglets. Based on the characteristics of PTD as an immunomodulator, we speculate that this may be due to the improvement of immune function and intestinal health.

Pidotimod promotes various aspects of the specific and non-specific immunity of the host (Tian and Zeng, 2005). For instance, PTD (0.5 g/l) promotes growth, lymphocyte proliferation, antibody production and increases the CD4/CD8 cell ratio also in poultry (Qu et al., 2017). In addition, PTD enhances the production of IL-2 in lymphocytes (Chiarenza et al., 1994) as well as up-regulates the expression of IL-8 level in individuals with Tourette's syndrome ( $\mathrm{Li}$ et al., 2015). IL-6 regulates chronic inflammation (Murakami et al., 2019). Together with IL-6, IFN- $\gamma$ and IL-8 are a predominant proinflammatory cytokines (Zelová and Hošek, 2013; Kim and Moudgil, 2017). However, the up-regulated IL-8 in another study was not found this time, and down-regulated pro-inflammatory cytokines including IL-6 and IFN- $\gamma$ were observed. It might be that no inflammation occurred in our research because there was no infection by a pathogen. Pathogen infection tends to stimulate the production of pro-inflammatory cytokines including IL-8, IL-6 leading to a generation of an inflammatory response (de Jong et al., 2006; Kobasa et al., 2007). In a previous study, PTD up-regulated the expression of IL-8 and IL-6, thereby enhancing the activity of the immune system for a long time (Esposito et al., 2015; Trabattoni et al., 2017). In addition, our PTD treatment increased serum levels of IL-10. Given that IL-10 and IL-2 modulate anti-inflammation
(Wojdasiewicz et al., 2014), PTD can inhibit inflammatory responses by regulating the expression of both pro-inflammatory and anti-inflammatory cytokines. This result was also observed in a previous study by Ucciferri et al. (2020), who also found a decreased level of pro-inflammatory cytokines in individuals infected with HIV. This implies that PTD exerts an anti-inflammatory effect in piglets through down-regulated pro-inflammatory cytokines and up-regulated anti-inflammation cytokines.

Besides being sites for digestion and food absorption, the intestines are the largest immune organs in the body. Meanwhile, the physiological functions of the intestines are based on the morphological integrity of the organ. Damage to the intestinal barrier disrupts their defence functions (Caramia et al., 1994). The intestinal epithelium is the first line of defence of the intestinal mucous system. It is comprised of connected tight junction (TJ) proteins which form a physical barrier against the entry of pathogens. The TJ is composed of occludin, claudins and ZO-1 proteins located in the cell. These three proteins are tightly bound together to form a TJ barrier that prevents the entry of antigenic substances (Swamy et al., 2010). In this study, we found that PTD improved the morphological structure of intestinal mucosa by strengthening the physical barrier at $\mathrm{TJ}$ and reducing swelling as well as cavitation of epithelial cells. Defects in barrier integrity caused chronic inflammation of the intestine (Suzuki, 2020), the maintenance and protection of the TJ barrier could be effective for preventing diseases and promoting growth. In addition, PTD significantly up-regulated the expression of jejunal TJ proteins such as occludin and ZO-1. TNF- $\alpha$ and interleukins may affect paracellular permeability by altering the expression of proteins in the TJ, thereby disrupting the barrier for antigens and bacteria (Camilleri et al., 2012). In this study, the reducing levels of pro-inflammatory cytokines were found. Combined with the up-regulated expression of TJ proteins, we thought that PTD could improve intestinal barrier function by anti-inflammatory effect, thereby enhancing the intestinal anti-infection ability which is beneficial to maintain intestinal health. Overall, PTD can effectively improve the intestinal epithelium integrity and intestinal physical barrier function in piglets.

The animal intestinal microbial communities are interconnected, with some exhibiting antagonistic relationships. The dynamic intestinal micro- 
bial interactions affect the health of the host (Duncan and Edberg, 1995). We found Firmicutes and Bacteroidetes to be the predominant bacteria in the pig gut $(>90 \%)$, which is consistent with the previvous study (Kim et al., 2011). Pidotimod significantly reduced the relative abundance of the Spirochaetes, Verrucomicrobia, Succinispira and Treponema but increased those of the Lactobacillus and Collinsella. Research shows that colonization of Spirochetes in the large intestine can cause several intestinal diseases in animals (Shears, 1997). On the other hand, a high relative abundance of Verrucomicrobia in the intestines induced Fusobacterium nucleatum infection and promoted colonic tumorigenesis ( $\mathrm{Li}$ et al., 2017). Also, certain Treponema species are associated with digital dermatitis in dairy cattle (Beninger et al., 2018). Reducing the relative abundance of these harmful bacteria PTD can improve microbial composition. It was observed that the digestibility of neutral detergent fibre positively correlated with the abundance of Collinsella (Niu et al., 2019). Consequently, higher intestinal Lactobacillus and Collinsella levels could positively improve the intestinal health of animals. It is worth mentioning that intestinal microbes have been reported to alter epithelial permeability indirectly through effects on host immune cells and the release of cytokines, which can regulate barrier function (Camilleri et al., 2012). Lactobacillus was associated with barrier function integrity in vivo and in vitro (Anderson et al., 2010; Mujagic et al., 2017). Thus, the increased Lactobacillus level after PTD treatment in our finding might contribute to intestinal health. Combined with previous research, it has been demonstrated that PTD improves the composition of intestinal microbiota by reducing the relative abundance of these harmful microbes and increasing the relative abundance of these beneficial microbes.

\section{Conclusions}

Pidotimod at a dose of $50 \mathrm{mg} / \mathrm{kg}$ may play a beneficial role in improving growth performance and intestinal health by enhancing immune function, intestinal epithelial barrier, and regulating intestinal ones.

\section{Acknowledgments}

The present work was supported by the grants from the planning subject of 'the Twelfth Five-year-plan' in National Science and Technology for the Rural Development in China (grant no.
2013BAD10B03). The authors thank Baikui Wang for the microbial analysis of the manuscript.

\section{Conflict of interest}

The authors declare that there is no conflict of interests.

\section{References}

Amadori M., Zanotti C., 2016. Immunoprophylaxis in intensive farming systems: the way forward. Vet. Immunol. Immunopathol. 181, 2-9, https://doi.org/10.1016/j.vetimm.2016.02.011

Anderson R.C., Cookson A.L., McNabb W.C., Kelly W.J., Roy N.C., 2010. Lactobacillus plantarum DSM 2648 is a potential probiotic that enhances intestinal barrier function. FEMS Microbiol. Lett. 309, 184-192, https://doi.org/10.1111/j.15746968.2010.02038.x

Beninger C., Naqvi S.A., Naushad S., Orsel K., Luby C., Derakhshani H., Khafipour E., De Buck J., 2018. Associations between digital dermatitis lesion grades in dairy cattle and the quantities of four Treponema species. Vet. Res. 49, 111, https://doi.org/10.1186/s13567-018-0605-z

Camilleri M., Madsen K., Spiller R., Van Meerveld B.G., Verne G.N., 2012. Intestinal barrier function in health and gastrointestinal disease. Neurogastroenterol. Motil. 24, 503-512, https://doi. org/10.1111/j.1365-2982.2012.01921.x

Caporaso J.G., Kuczynski J., Stombaugh J. et al., 2010. QIIME allows analysis of high-throughput community sequencing data. Nat. Methods 7, 335-336, https://doi.org/10.1038/nmeth.f.303

Capsoni F., Minonzio F., Ongari A.M., Girardello R., Zanussi C., 1992. Evaluation of the kinetics of the immunomodulating activity of pidotimod on human phagocytes. Pharmacol. Res. 26, Suppl. 2, 172-173, https://doi.org/10.1016/1043-6618(92)90650-Z

Caramia G., Clemente E., Solli R., Mei V., Cera R., Carnelli V., Venturoli V., Corsini A., 1994. Efficacy and safety of pidotimod in the treatment of recurrent respiratory infections in children. Arzneimittel-Forschung 44, 1480-1484

Chiarenza A., lurato M.P., Barbera N., Lempereur L., Cantarella G., Scapagnini U., Scapagnini G., Bernardini R., 1994. Effects of pidotimod on the immune and the neuroendocrine system in the aging rat. Arzneimittel-Forschung 44, 1437-1440

Clemente E., Solli R., Mei V., Cera R., Caramia G., Carnelli V., Ruffini E., Venturoli V., Corsini A., 1994. Therapeutic efficacy and safety of pidotimod in the treatment of urinary tract infections in children. Arzneimittel-Forschung 44, 1490-1494

Coppi G., Amico-Roxas M., Berte F., Bussi R., Gnemi P., Harling R., Mailland F., Manzardo S., Massey J., Spencer-Briggs D., 1994. Toxicological evaluation of pidotimod. ArzneimittelForschung 44, 1448-1453

Coppi G., Silingardi S., 1994. Pharmacokinetics of pidotimod in rats and dogs. Arzneimittel-Forschung 44, 1460-1464

de Jong M.D., Simmons C.P., Thanh T.T. et al., 2006. Fatal outcome of human influenza a (H5N1) is associated with high viral load and hypercytokinemia. Nat. Med. 12, 1203-1207, https://doi. org/10.1038/nm1477

Duncan H.E., Edberg S.C., 1995. Host-microbe interaction in the gastrointestinal tract. Crit. Rev. Microbiol. 21, 85-100, https:// doi.org/10.3109/10408419509113535 
Esposito S., Garziano M., Rainone V. et al., 2015. Immunomodulatory activity of pidotimod administered with standard antibiotic therapy in children hospitalized for community-acquired pneumonia. J. Transl. Med. 13, 288, https://doi.org/10.1186/ s12967-015-0649-z

Ferrario B.E., Garuti S., Braido F., Canonica G.W., 2015. Pidotimod: the state of art. Clin. Mol. Allergy 13, 8, https://doi.org/10.1186/ s12948-015-0012-1

Fu L.Q., Fu A.K., Li L., Hu S.L., Li W.F., 2016. Detection and stability of pidotimod by high performance liquid chromatography. Chin. J. Prev. Vet. Med. 38, 381-384, https://doi.org/10.3969/j. issn.1008-0589.2016.05.09

Fulde M., Hornef M.W., 2014. Maturation of the enteric mucosal innate immune system during the postnatal period. Immunol. Rev. 260, 21-34, https://doi.org/10.1111/imr.12190

Giagulli C., Noerder M., Avolio M., Becker P.D., Fiorentini S., Guzman C.A., Caruso A., 2009. Pidotimod promotes functional maturation of dendritic cells and displays adjuvant properties at the nasal mucosa level. Int. Immunopharmacol. 9, 1366-1373, https://doi.org/10.1016/j.intimp.2009.08.010

Kamada N., Chen G.Y., Inohara N., Nunez G., 2013. Control of pathogens and pathobionts by the gut microbiota. Nat. Immunol. 14, 685-690, https://doi.org/10.1038/ni.2608

Kim E.Y., Moudgil K.D., 2017. Immunomodulation of autoimmune arthritis by pro-inflammatory cytokines. Cytokine $98,87-96$, https://doi.org/10.1016/j.cyto.2017.04.012

Kim H.B., Borewicz K., White B.A., Singer R.S., Sreevatsan S., Tu Z.J., Isaacson R.E., 2011. Longitudinal investigation of the age-related bacterial diversity in the feces of commercial pigs. Vet. Microbiol. 153, 124-133, https://doi.org/10.1016/j. vetmic.2011.05.021

Kobasa D., Jones S.M., Shinya K. et al., 2007. Aberrant innate immune response in lethal infection of macaques with the 1918 influenza virus. Nature 445, 319-323, https://doi. org/10.1038/nature05495

Li E., Ruan Y., Chen Q., Cui X., Lv L., Zheng P., Wang L., 2015. Streptococcal infection and immune response in children with Tourette's syndrome. Child. Nerv. Syst. 31, 1157-1163, https://doi.org/10.1007/s00381-015-2692-8

Li Y.L., Fu A.K., Chen H.L., Li W.F., Fu L.Q., 2016. Potentiating effect of pidotimod on immune responses of chickens to live attenuated Newcastle disease vaccines. Ital. J. Anim. Sci. 15, 536-544, https://doi.org/10.1080/1828051X.2016.1218305

Li J., Yu Y., Jiang X., Lu Y., Tian Z., 2017. Fusobacterium nucleatum prompts colonic tumorigenesis in mice and its potential mechanism. Chin. J. Gastroenter. 22, 396-401, https://doi. org/10.3969/j.issn.1008-7125.2017.07.003

Mahashur A., Thomas P.K., Mehta P., Nivangune K., Muchhala S., Jain R., 2019. Pidotimod: In-depth review of current evidence. Lung India 36, 422-433, https://doi.org/10.4103/lungindia. lungindia_39_19

Migliorati G., D’Adamio L., Coppi G., Nicoletti I., Riccardi C., 1992. Pidotimod stimulates natural killer cell activity and inhibits thymocyte cell death. Immunopharmacol. Immunotoxicol. 14, 737-748, https://doi.org/10.3109/08923979209009231

Migliorati G., Nicoletti I., Delfino D., Maggioni A., Coppi G., Riccardi C., 1993. Pidotimod causes apoptotic cell death and inhibits the proliferative activity of YAC-1 tumor cells in vitro. Drugs Exp. Clin. Res. 19, Suppl., 1-7

Migliorati G., Nicoletti I., Riccardi C., 1994. Immunomodulating activity of pidotimod. Arzneimittelforschung 44, 1421-1424
Mujagic Z., de Vos P., Boekschoten M.V., Govers C., Pieters H.H., de Wit N.J.W., Bron P.A., Masclee A.A., Troost F.J., 2017. The effects of Lactobacillus plantarum on small intestinal barrier function and mucosal gene transcription; a randomized double-blind placebo controlled trial. Sci. Rep. 7, 40128, https://doi.org/10.1038/srep40128

Murakami M., Kamimura D., Hirano T., 2019. Pleiotropy and specificity: insights from the interleukin 6 family of cytokines. Immunity 50, 812-831, https://doi.org/10.1016/j. immuni.2019.03.027

Niu Q., Li P., Hao S., Kim S., Du T., Hua J., Huang R., 2019. Characteristics of gut microbiota in sows and their relationship with apparent nutrient digestibility. Int. J. Mol. Sci. 20, 870, https://doi.org/10.3390/ijms20040870

NRC (National Research Council), 2012. Nutrient Requirements of Swine. $11^{\text {th }}$ Revised Edition. The National Academies Press. Washington, DC (USA), https://doi.org/10.17226/13298

Puggioni F., Alves-Correia M., Mohamed M.F. et al., 2019. Immunostimulants in respiratory diseases: focus on Pidotimod. Multidiscip. Resp. Med. 14, 31, https://doi. org/10.1186/s40248-019-0195-2

Qu S., Dai C., Qu M., Zhang R., Wang C., Cui L., Hao Z., 2017. Effects of pidotimod soluble powder and immune enhancement of Newcastle disease vaccine in chickens. Immunol. Lett. 187, 14-18, https://doi.org/10.1016/j.imlet.2017.05.001

Riboldi P., Gerosa M., Meroni P.L., 2009. Pidotimod: a reappraisal. Int. J. Immunopathol. Pharmacol. 22, 255-262, https://doi. org/10.1177/039463200902200201

Shears P., 1997. Intestinal Spirochaetes in domestic animals and humans. Trans. R. Soc. Trop. Med. Hyg. 91, 730, https://doi. org/10.1016/S0035-9203(97)90547-2

Schneider C.A., Rasband W.S., Eliceiri K.W., 2012. NIH Image to ImageJ: 25 years of image analysis. Nat. Method. 9 , 671-675, https://doi.org/10.1038/nmeth.2089

Suzuki T., 2020. Regulation of the intestinal barrier by nutrients: The role of tight junctions. Anim. Sci. J. 91, e13357, https://doi. org/10.1111/asj.13357

Swamy M., Jamora C., Havran W., Hayday A., 2010. Epithelial decision makers: In search of the 'epimmunome'. Nat. Immunol. 11, 656-665, https://doi.org/10.1038/ni.1905

Tian X.P., Zeng X.F., 2005. A new synthetic immunomodulator pidotimod. Chin. New Drugs J. 1, 111-114

Trabattoni D., Clerici M., Centanni S., Mantero M., Garziano M., Blasi F., 2017. Immunomodulatory effects of pidotimod in adults with community-acquired pneumonia undergoing standard antibiotic therapy. Pulm. Pharmacol. Ther. 44, 24-29, https://doi.org/10.1016/j.pupt.2017.03.005

Ucciferri C., Falasca K., Reale M., Tamburro M., Auricchio A., Vignale F., Vecchiet J., 2020. Pidotimod and immunological activation in individuals infected with HIV. Curr. HIV Res. 18, 1, https://doi.org/10.2174/1570162X18666210111102046

Wang S., Charbonnier L.M., Rivas M.N., Georgiev P., Ning L., Gerber G., Bry L., Chatila T.A., 2015. MyD88 adaptordependent microbial sensing by regulatory $T$ cells promotes mucosal tolerance and enforces commensalism. Immunity 43, 289-303, https://doi.org/10.1016/j.immuni.2015.06.014

Wojdasiewicz P., Poniatowski Ł.A., Szukiewicz D., 2014. The role of inflammatory and anti-inflammatory cytokines in the pathogenesis of osteoarthritis. Mediat. Inflamm. 2014, 561459, https://doi.org/10.1155/2014/561459 
Wu F., Ding X.Y., Li X.H., Gong M.J., An J.Q., Huang S.L., 2020. Correlation between elevated inflammatory cytokines of spleen and spleen index in acute spinal cord injury. J. Neuroimmunol. 344, 577264, https://doi.org/10.1016/j. jneuroim.2020.577264

Xu X., Gong L., Wang B., Wu Y. et al., 2018. Glycyrrhizin attenuates Salmonella enterica serovar typhimurium infection: new insights into its protective mechanism. Front. Immunol. 9, 2321, https://doi.org/10.3389/fimmu.2018.02321

Zelová H., Hošek J., 2013. TNF-a signalling and inflammation: interactions between old acquaintances. Inflamm. Res. 62, 641-651, https://doi.org/10.1007/s00011-013-0633-0
Zhong T.M., Mao F.J., Sun D.H., 2005. A test on medicine precaution and treatment of diarrhea of early-weaned piglets. Acta Agric. Zhejiangensis 17, 90-93

Zhong J.F., Wu W.G., Zhang X.Q., Tu W., Liu Z.X., Fang R.J., 2016. Effects of dietary addition of heat-killed Mycobacterium phlei on growth performance, immune status and anti-oxidative capacity in early weaned piglets. Arch. Anim. Nutr. 70, 249-262, https://doi.org/10.1080/1745039X.2016.1183365 